\title{
Assessment of fracture healing properties of lovastatin loaded nanoparticles: preclinical study in rat model
}

\author{
Peng Zhu', Guiyun Huang², Bing Zhang1', Wenzhi Zhang³ ${ }^{3}$ Minyan Dang ${ }^{3}$ \\ and Zhang Huang 4 区 \\ 'Department of Orthopedics, The Second Affiliated Hospital of Xi'an Medical University, Baqiao District, Xi'an, Shaanxi Province, 710038, China; \\ 2Department of Orthopedics, Jiyang Public Hospital of Shandong Province, Jinan, Shandong, 251400, China; 3Innoscience Research SdnBhd, \\ Jalan USJ 25/1, 47650 Subang Jaya, Selangor Malaysia; “Department of Orthopaedics, Hanzhong People's Hospital, Hantai District, Hanzhong \\ City, Shaanxi Province, 723000, China
}

Bone fracture, being mainly caused by mechanical stress, requires special and quick attention for a rapid healing. The study presented here aims at formulating nanoparticulate system to overcome the solubility issues of lovastatin. The lovastatin nanoparticles were successfully prepared by ionotropic gelation method using chitosan and tri-polyphosphate as polymers. Thus prepared nanoparticles were found to be smooth and spherical with average particle size of $87 \mathrm{~nm}$ and encapsulation efficiency of $86.5 \%$. The in-vitro drug release was found to be almost $89.6 \%$ in the first 360 minutes. Artificial fracture was produced in female Wistar rats at right leg using fracture apparatus. After administration of lovastatin nanoparticles or saline solution, the respective groups were observed for various parameters. The X-ray imaging showed that lovastatin accelerated bone healing, compared to control. The growth of animals was not hampered by lovastatin by any means. The radiographic examination confirmed a role of lovastatin in increasing bone density. The histological study showed the broken, proliferated and discontinued trabecullae in the control, while at the same time point, the normal, thick, continuous and connected trabecullae were observed in animals administered with lovastatin nanoparticles. The biomechanical studies showed high breaking resilience and minimum bone brittleness in animals injected with lovastatin nanoparticles. Considering these observations we state that lovastatin helps in rapid bone healing after fracture via increasing the bone density.

Key words: fracture healing, lovastatin, nanoparticles, rat model

Received: 23 October, 2018; revised: 02 November, 2018; accepted: 16 January, 2019; available on-line: 11 March, 2019

e-mail: yinuo1601@sina.com

Abbreviations: CS-LST, Lovastatin loaded chitosan nanoparticles; CS, chitosan; DLS, dynamic light scattering; DR, drug release; E.E., encapsulation efficiency; MWCO, molecular weight cut off; PBS, phosphate buffered saline; PDI, polydispersity index; TPP, tripoly phosphate

\section{INTRODUCTION}

Bone fracture is a discontinuity in the bone. The leading causes of bone fracture are mechanical impact or stress. The other reasons include osteoporosis, age, some type of cancers and osteogenesis imperfecta (brittle bone disease). The incidence of fracture increases with age in both men and women, but this proportion is greater among women. With the worldwide increase in traffic, the transportation utilities have become the leading cause of injuries (Altundag et al., 2004), and bone fracture is one of the most common form of all the injuries. It has burdened the society with the increase in the treatment costs, a decrease in productivity, and deterioration of the overall quality of life. To reduce morbidity and mortality, it is vital to accelerate the fracture healing.

Bone fracture treatment often involves setting and immobilizing bones to give time to heal. Fracture healing process is complex, both on intracellular and extracellular level, and involves three main stages: reactive, reparative and remodeling (Grundnes et al., 1993). To initiate healing, the growth factors such as osteocalcin, bone morphogenetic protein (BMP)-2, vascular endothelial growth factor (VEGF)- $\alpha$, bone sialoprotein, Runt-related transcription factor (Runx)-2, and FGF-23 start to accumulate at the site of fracture. They promote proliferation of undifferentiated cells and secretion of cartilagespecific matrix, and subsequently, cartilage formation and osteogenesis (Einhorn et al., 1995; Hausman et al., 2001). As the healing progresses, the stem cells differentiate into either osteoblasts or chondrocytes to form soft callus, which fills in the gap between the bone fragments. As the time passes, the soft callus converts into hard callus by the process of ossification. Calcification of the cartilage occurs at the interface between the maturing chondrocyte and the newly forming bone as a result of chondrocyte apoptosis (Einhorn et al., 1998).

Statins have been found to trigger BMP2 expression in bone cells and thus accelerate healing of bone fractures (Hadjiargyrou et al., 2002). For osteogenesis, statin acts by (activating?) HMG-CoA reductase in mevalonate pathway. It also acts by antagonizing osteoclasts via increasing expression of osteoprotegerin, (inhibiting?) the $\mathrm{NF}-x \mathrm{~B}$ ligand (RANKL)-osteoprotegerin (OPG) pathway (Gerstenfeld et al., 2003). Lovastatin is widely used to treat patients with moderate hypercholesterolemia; it acts by reducing the formation of cholesterol (Giannotti et al., 2003). Apart from this, the use of lovastatin in the treatment of bone fractures and inflammatory diseases is being investigated. Simvastatin, when administered orally in high doses to mice with femoral injury, promoted the healing of fractures (Abdul Majeed et al., 2012). At a cholesterol-lowering dose, only a minor fraction of statins can reach the fracture site. However, when given in high doses, the statins can cause adverse effects such as liver failure, kidney disease and rhabdomyolysis. A preclinical study showed that administering statins by routes other than oral can increase bone resorption. To get a local effect of fracture healing using a low dose of statin, it is necessary to administer the drug to the fracture site (Claes et al., 2012) 
A large proportion of the developed (?) drugs has low solubility and/or oral bioavailability. Lovastatin, belonging to class II of the biopharmaceutical classification system, also has a very low water solubility, and hence is poorly absorbed from the gastrointestinal tract. Solubility and bioavailability of such hydrophobic drugs can be increased by creating nanoparticle formulations with high surface to volume ratios. Targeted delivery systems deliver and for a long time maintain the maximum drug concentration at the bone fracture site, as they release the drug at a constant rate. Using targeted delivery system, the adverse effects associated with the use of high doses of lovastatin may be avoided, while the bioavailability may be increased to promote fracture healing. Nanoparticle drug delivery system is one of the novel approaches for targeted drug delivery to treat various diseases and improve the bioavailability, stability, and performance of the drug. Lovastatin nanoparticles can be used in targeted drug delivery to the bone fracture site, eliminating various potential barriers and reducing the dose-dependent side effects. Lovastatin nanoparticles were also shown to enhance fracture healing and biomechanical properties of the bone (Abdul Majeed et al., 2012; McAnally et al., 2007).

A wide range of natural and synthetic polymers have been tested for the synthesis of nanoparticles, and among them chitosan has proven to be a promising polymer for the synthesis of targeted drug delivery system (Abdul Majeed et al., 2012; Gupta et al., 2006). Chitosan is a natural polysaccharide that can be synthesized from N-deacetylation of chitin, a biopolymer extracted from the shells of crustaceans such as crabs, shrimps and lobsters. Chemically, chitosan is a copolymer of $\mathrm{N}$-acetyl glucosamine and $\beta$-glucosamine moieties and may have varied molecular weights depending on the degree of acetylation (Bhattarai et al., 2006). Chitosan nanoparticles can be prepared by a simple, cost effective and non-toxic ionic gelation method using the TPP anions. Moreover, chitosan has some therapeutic properties itself, such as immunomodulating, antimicrobial and wound healing (Agnihotri et al., 2004; Mohammadpour Dounighi et al., 2004).

In the presented study, the lovastatin nanoparticles were formulated from chitosan polymer by ionotropic gelation method and administered in experimental rat model to further test their fracture healing properties.

\section{MATERIALS AND METHODS}

Materials. Lovastatin was a kind gift from Shouguang Fukang Pharmacy Factory (Shandong, China). Chitosan (CS), tripolyphosphate (TPP) and acetic acid were purchased from Sigma Aldrich, USA.

\section{Methods}

Preparation of lovastatin loaded chitosan nanoparticles. Ionotropic gelation method was used for preparation of nanoparticles of chitosan. Chitosan nanoparticles were synthesized via the ionotropic gelation (Gan et al., 2007) of chitosan with TPP anions. Chitosan was dissolved in acetic aqueous solution at various concentrations $(1,2,3 \mathrm{mg} / \mathrm{ml})$. The concentration of acetic acid in aqueous solution was 1.5 time higher than that of chitosan. The TPP solution of concentration $1 \mathrm{mg} / \mathrm{ml}$ was prepared in double distilled water. To prepare the nanoparticles, $5 \mathrm{ml}$ of the chitosan solution was added drop wise to $2 \mathrm{ml}$ of TPP solution under magnetic stirring (1000 rpm, 1 hour) at room temperature, until the opaque suspension was obtained. Then the prepared nanoparticles were separated by centrifuging the suspension at $20000 \times g, 14^{\circ} \mathrm{C}$ for 30 minutes, and then freezedried and stored at $5 \pm 3^{\circ} \mathrm{C}$. The mass of freeze-dried nanoparticles was also determined. The lovastatin loaded nanoparticles (CS-LST) were characterized and optimized using the methods described in (Gan Q et al., 2007).

Evaluation of nanoparticles. Particle size determination. For the morphological measurements of nanoparticles, the transmission electron microscopy (TEM) was used. TE microscope works at a distance of 8.6$8.8 \mathrm{~mm}$ with accelerating voltage of $1.0 \mathrm{kV}$. The particle size, its distribution and zeta potential were measured by Zetasizer (Malvern/DTS 4.1), which works using the dynamic light scattering (DLS) technique.

In order to make measurements, the samples were suspended in water by rapid mixing and the readings were recorded in triplicate.

In-vitro drug release. The study of release characteristics was performed for both plain and nanoparticleencapsulated drug. Molecular weight cut off (MWCO) dialysis membrane with sufficiently large pores for allowing the passage of lovastatin was used. About $100 \mathrm{mg}$ of drug or suspended nanoparticles with the encapsulated drug $100 \mathrm{mg}$ equivqlent were put into the dialysis bag, which was then sealed at both ends and put in $1000 \mathrm{ml}$ of $0.1 \mathrm{M}$ phosphate buffer of $\mathrm{pH}$ 7.4. The USP type XXII (rotating paddle) apparatus was used in the study. It was maintained at $37 \pm 2^{\circ} \mathrm{C}$ and 100 and $200 \mathrm{rpm}$ rotor speed. At the time points of $0,5,15,30,60,120$ and $360 \mathrm{~min}, 5 \mathrm{ml}$ aliquots were taken for testing, and replaced with the same amount of buffer solution. The UV spectrophotometer was used to analyze the sample (Rezaei Mokarram et al., 2006).

Drug encapsulation. Lovastatin was separated from the nanoparticle suspension by centrifugation at 20000 $\mathrm{RPM}$ and $40^{\circ} \mathrm{C}$ for $30 \mathrm{~min}$. The supernatant was collected and the concentration of free lovastatin originating from nanoparticles was measured by UV spectrophotometer at $525 \mathrm{~nm}$. The encapsulation efficiency (E.E.) of the nanoparticles was calculated by using the following equation:

$\%$ E.E $=[($ Free drug $/$ Total amount of lovastatin $) /$ Total weight of nanoparticles] $\times 100$.

In vivo rat fracture model. Female Wistar rats of around 3 weeks of age and the mass of 65 grams were used in this study. Animals were divided into two groups $(n=7)$. Pinned closed transverse rat femoral model depicted by Bonnarens et al. was used for the study, because of its reproducibility and uniformity The rats were kept two per cage with a 12-hour light-dark cycle and allowed free cage activity and food and water ad libitum. After one week of acclimatization, the rats were assigned randomly into 2 groups with 7 rats per group and a single dose of $50 \mathrm{ml}$ solution of phosphate-buffered saline (PBS) (Group I) or nanoparticulate formulation containing lovastatin at a concentration of $4 \mathrm{ng} / \mathrm{ml}$ (Group II) was administered to the rats after fracture to the right leg of each animal. The animals were observed for fracture healing at predetermined time intervals of 2 weeks, 6 weeks, 3 months and 6 months. All animal experiments were performed in agreement with the animal ethical committee of Second Affiliated Hospital of Xi'an Medical University with decision/ ethical no. 20181102.

Artificial fracturing of femoral shaft. Fracture was produced in both groups of animals by using fracture apparatus. Animals were anesthetized and an incision was made in middle of the patella of right leg. Stainless steel 
Table 1. The coded values for the response for formulation of lovastatin nanoparticles using factorial design, where -1 is lowest concentration and +1 is highest concentration of the polymers.

\begin{tabular}{lll}
\hline Independent Variable & Low level $(-1)$ & High level $(+1)$ \\
\hline $\mathrm{A}=$ CS Conc. (mg) & 100 & 200 \\
\hdashline $\mathrm{B}=$ TPP Conc. (\%) & 0.5 & 0.75 \\
\hline C=Tween 80 Conc. (\%) & 0.5 & 1
\end{tabular}

Dependent Variables

$Y 1=E . E$.

Y2=Drug release

Kirschner wires $(1.1 \mathrm{~mm}$ diameter $)$ were inserted in a backward direction through the femoral shaft and pulled out of the greater trochanter. X-rays were obtained every week after fracture. Animals were kept in the cages and had food and water ad libitum. At the end of the 4th week, the animals were euthanized by cervical dislocation or by injecting ketamine at a dose of $100 \mathrm{mg} /$ $\mathrm{kg}$ of body weight. Fractured femurs were removed and stored in $0.145 \mathrm{M} \mathrm{NaCl}$ at $-70^{\circ} \mathrm{C}$ for bio-mechanical test (Stuermer et al., 2006)

Growth. The rats were weighted and physically observed throughout the study period. Changes in body mass and femur length were recorded for all the groups at 2 nd and 4 th week and on a regular interval of 2 weeks.

Radiographic examination. Radiographic examination was done by using table top procedure. X-rays were taken every week. The exposure time for X-ray was 10 $\mathrm{ms}$ at $1 \mathrm{~m}$ distance.

Bone histology. At the end of the treatment period (4th week) three animals in each group were euthanized with intra-cardiac injection of Dolethal. The compact and spongy bone from the femur were then removed for histological evaluation and scanning electron microscopy. The spongy bone was isolated from radius and head of the femur and fixed in 4\% formaldehyde solution for 48 hours. The compact bone was demineralized by keeping in formic acid for 21 days. After washing in running water for $6 \mathrm{hrs}$, the sections of $7 \mathrm{um}$ of bone were taken and stained with hematoxylin and eosin as described in (Stuermer et al., 2006; Garrett et al., 2007).

Bone biomechanics. The isolated femurs were subjected to three-point bending. Each femur was positioned horizontally on the support rollers (which were $12 \mathrm{~mm}$ apart), so that the vertical, rounded indenter

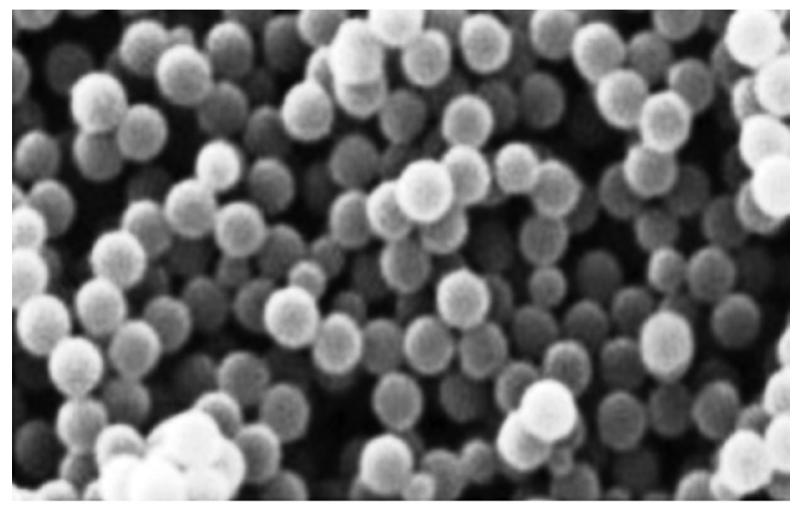

Figure 1. TEM image of nanoparticles.

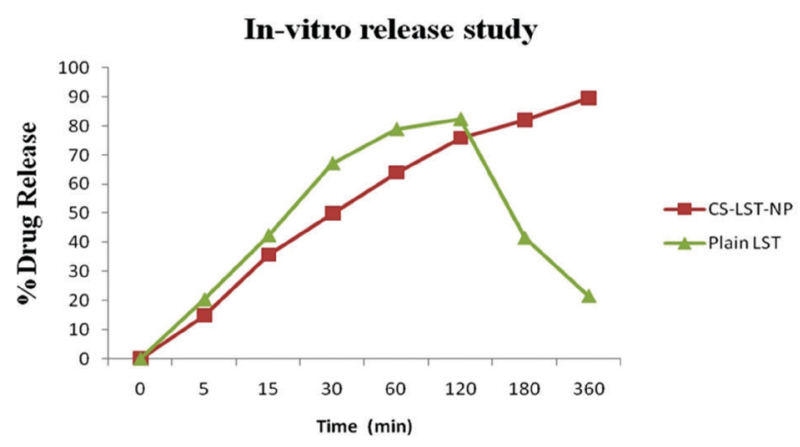

Figure 2. In vitro drug release analyzed with UV spectrophotometer.

pressed the callus with the medial side in front and the anterior side down (i.e., bending occurred about the medial-lateral axis). The force-displacement curve was recorded as the indenter traveled at $3 \mathrm{~mm} / \mathrm{min}$ into the callus. These data provided data on the following structural properties: breaking force (maximum load), stiffness (average slope of the linear portion of the curve before yielding), and work-to-fracture (area under the curve).

Statistical analysis. The statistical analysis was performed using the Design Expert software 6.0.8. The data obtained were presented as the mean \pm standard deviation. $p<0.05$ was considered statistically significant.

\section{RESULTS}

The lovastatin nanoparticles were formulated using polymers at various concentrations as shown in Table 1. Average size of lovastatin nanoparticle was found to be in the range of 60-109 $\mathrm{nm}$, as calculated using the transmission electron microscope method (Fig. 1). CS-LST (Chitosan Lovastatin) nanoparticles were obtained in the size range of 92.50 to $482.50 \mathrm{~nm}$ with poly dispersity index (PDI) from 0.120 to 0.384. TMZ loading resulted in a slight increase in the particle size. We observed that $90 \%$ of lovastatin has been released from chitosan nanoparticles within the first $360 \mathrm{~min}$ after administration. The release profile of lovastatin loaded nanoparticles (as shown in Fig. 2) exhibits initial burst release of $10.75 \%$ of lovastatin followed by slow gradual sustained release of $\sim 89.56 \%$. The percentage Encapsulation efficiency (\%E.E.) of nanoparticles in our study was found to be in the range of $79.41 \%$ to $89.56 \%$ as shown in Fig. 3. Healing of fracture in Group II rats is shown in Fig. 4. The $\mathrm{X}$ ray images presented in Fig. 4 confirm that lovastatin helps greatly in fracture healing.
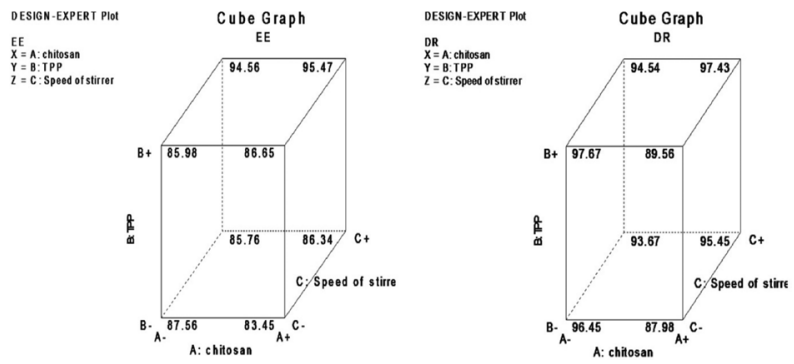

Figure 3. Cube graphs showing effect of chitosan and TPP concentration together with the speed of stirrer on the encapsulation efficiency and \% drug release. 


\section{GROUP I}

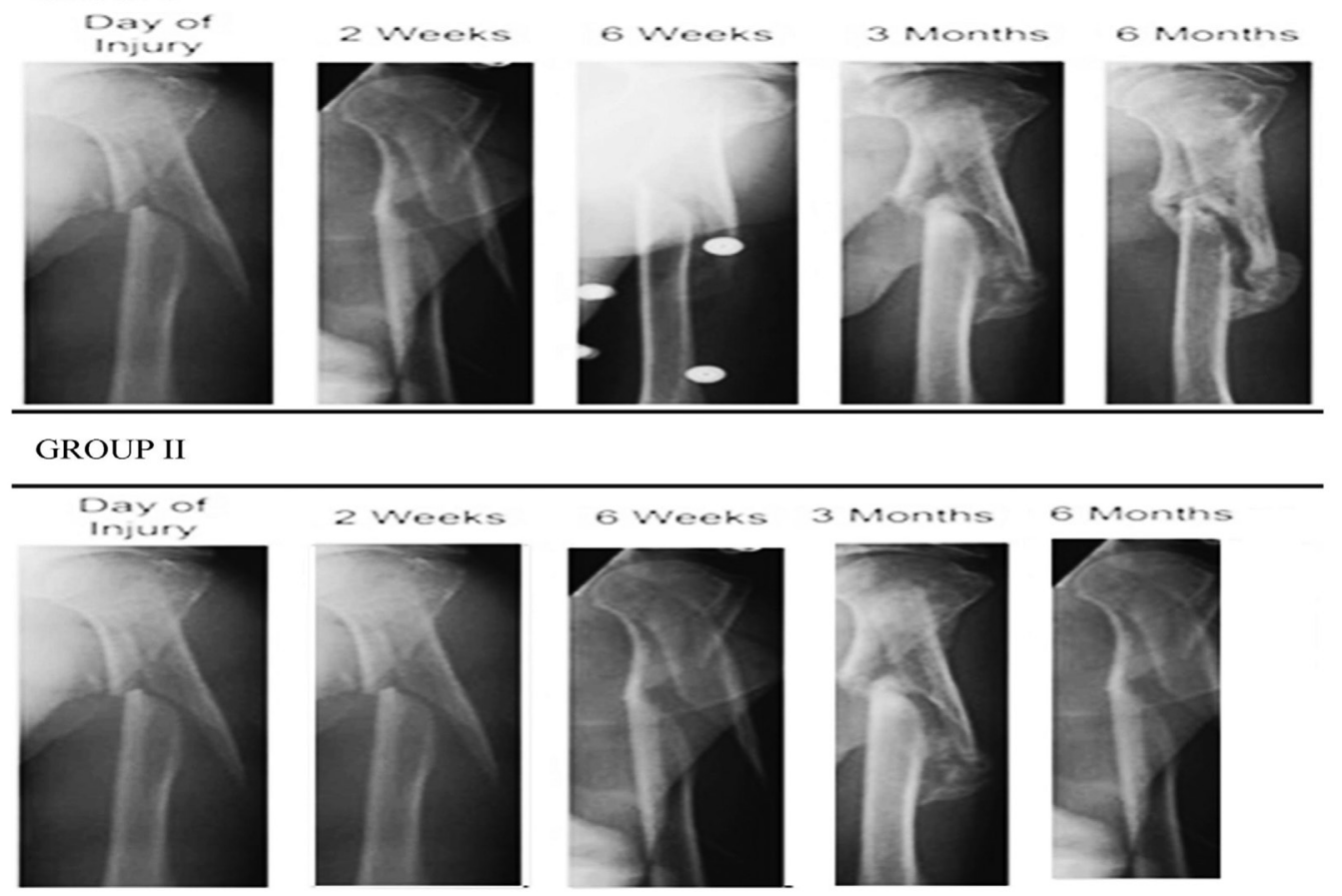

Figure 4. X-ray images showing fracture healing in rats over time.

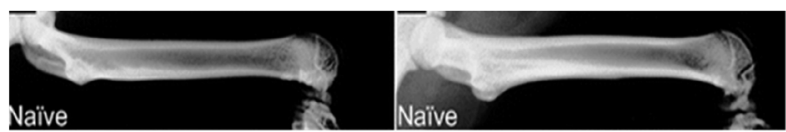

Figure 5. Radiographs of the femurs taken on (give a timepoint here) after fracture. Left side: femur from a group I rat; right side: femur from a group II rat.
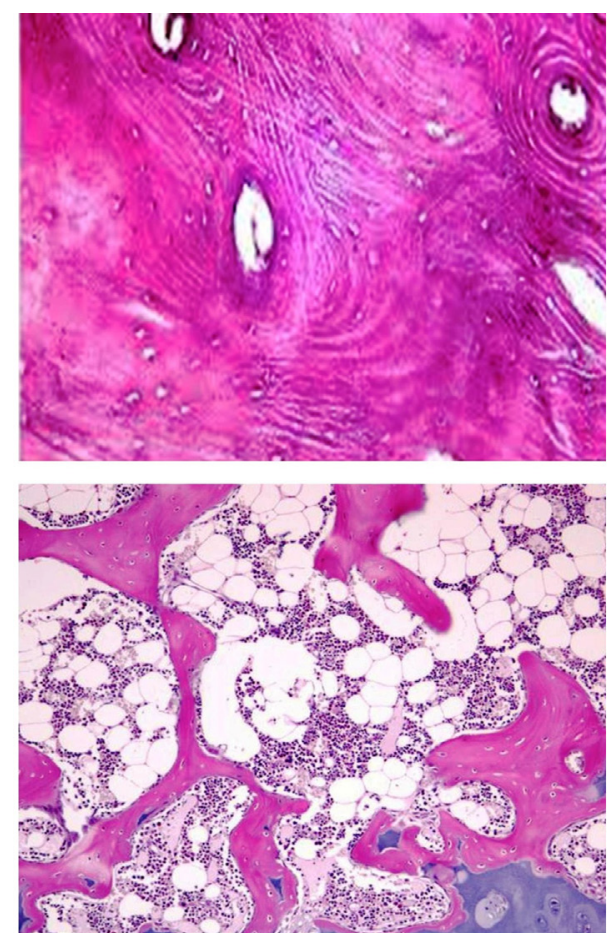

Figure 6. Femoral head showing continuous, thick trabecullae in group II rats (upper picture) and broken and elongated trabecullae in group I (bottom picture).
Table 2. Actual values in factorial design with their respective concentration in formulation.

\begin{tabular}{llllll}
\hline \multirow{2}{*}{ Batches } & \multicolumn{3}{l}{ Factor } & \multicolumn{5}{l}{ Response } \\
\cline { 2 - 6 } & A & B & C & Y1 & Y2 \\
\hline F1 & -1 & -1 & -1 & 85.93 & 235.54 \\
\hdashline F2 & +1 & -1 & +1 & 78.80 & 334.56 \\
\hline F3 & -1 & +1 & -1 & 90.92 & 167.34 \\
\hline F4 & +1 & +1 & +1 & 97.85 & 92.50 \\
\hline F5 & +1 & +1 & -1 & 92.87 & 110.45 \\
\hline F6 & -1 & +1 & +1 & 91.67 & 135.76 \\
\hdashline F7 & -1 & -1 & -1 & 75.32 & 482.50 \\
\hdashline F8 & +1 & -1 & +1 & 93.26 & 98.78 \\
\hline
\end{tabular}

Table 3. Biomechanical data showing structural and material properties of the femur.

\begin{tabular}{lll}
\hline & Group I & Group II \\
\hline Structural stiffness (N/mm) & $31.2 \pm 1.7$ & $44.9 \pm 1.43$ \\
\hline Breaking strength (MPa) & $10.2 \pm 2.67$ & $169.2 \pm 3.23$ \\
\hline Brittleness (\% elasticity) & $95.7 \pm 1.54$ & $74.9 \pm 1.3$ \\
\hline
\end{tabular}

After 2 weeks, the group II rats had higher body mass gain than the rats in group I. The average body mass of the animals in group I was $64.5 \mathrm{~g}$, while it was $68.7 \mathrm{~g}$ in group II. The values of gain of mass in group II were statistically significant. We have not observed any adverse effects of nanoparticles on weight gain in both groups. Radiographs of the animals in each group were taken on a weekly basis using the table top procedure. At the end of the treatment, the X-rays of the isolated femurs from animal bodies were taken. We found that in group I animals (Fig. 5: left) the radius was radiolucent, 
with a very thin cortex and an abnormal density of the marrow cavity, whereas femurs in group II (Fig. 5: right) were normal.

In histopathological study the cancellous bone, wing of ilium, and femur head were assessed. In Group I, the trabecullae wing was found to be broken and proliferated with discontinuity of trabecullae in ilium wing and femoral head. At the same time, in group II, normal, thick bone trabeculae were continuous and connected with iliac wing and femoral head (Fig. 6). Thickness of trabecullae was measured in all groups. Significant difference $(p<0.02)$ between first and second group was observed. Size of lacunae was larger in group I when compared with group II $(p<0.02)$.

Biomechanical properties of the previously fractured femurs were studied at week 4th (?) after the injury, and we found that structural stiffness and breaking strength is higher in group II compared to group I. Brittleness was higher in group I compared to group II (Table 2).

\section{DISCUSSION}

The lovastatin nanoparticles were formulated using polymers at various concentrations, as shown in $\mathrm{Ta}-$ ble 1 . The -1 sign in table 1 indicates the low concentration of polymer while +1 sign indicates the highest concentration used in formulation. F1 to F9 are the batch numbers allotted using various combinations of drug and polymers. The levels of independent and dependable variables are shown in Table 3. In this study, Malvern particle size analyzer (DVS) showed nanosized particles with a very narrow PDI. Also, as the concentration of the cross linking agent TPP increased, the particle size decreased significantly. The observed enhancement in the encapsulation efficiency (E.E.) was due to the chitosan which has higher ability of the ionic gel formation with the TPP that results in to increasing the E.E. The low E.E. was attributed to the smaller amount of CS and TPP used during the nanoparticles formulation. It was a general concept that the increased E.E may be due to the higher concentration of polymer relative to the amount of the drug used during the nanoparticles formulation. Higher concentration of the polymer forms the denser mass of the drug polymer dispersion, which helps to entrap the drug molecule in the nanoparticles. Nanoparticles prepared with a high concentration of the crosslinking agent showed increase in the E.E. The polynomial equation obtained for E.E. (Y1) is given by:

$\mathrm{Y} 1=+51.50+17.45 \mathrm{~A}+5.63 \mathrm{~B}+9.48 \mathrm{C}$

The increase in crosslinker density might prevent the leaching of drug molecule during the nanoparticles formation. The cube plots showing this interdependence are presented in Fig. 4. Our results confirmed the efficiency of lovastatin in improving of the healing of bone fracture. The effect of independent variables on the E.E. was found to be statistically significant $(p<0.03)$.

For the initial burst release pattern, the best fitted model is Higuchi model $(r=0.987)$, which indicates the diffusion-based drug release pattern. The semi-empirical Koremeyer-Peppas formula can describe the drug release mechanism in this study more precisely. The ' $n$ ' value of 0.84 suggests a non-fickian mechanism of drug release, i.e. drug release by diffusion and erosion. The polynomial equation obtained for DR (Y2) is given by:

$\mathrm{Y} 2=+49.70+15.30 \mathrm{~A}+3.43 \mathrm{~B}+6.42 \mathrm{C}$
The effect of independent variables on E.E. was found to be statistically significant $(p<0.02)$.

Lovastatin is an anti-hyperlipidemic agent used to treat hypercholesterolemia, which was found to exhibit anabolic actions on bone through the stimulation of BMP2 expression. The action of statin on bone formation is proposed to go via its activity on $\mathrm{HMG}-\mathrm{CoA}$ reductase in mevalonate pathway, also known as cholesterol biosynthetic pathway. Statin may also antagonize osteoclasts by increasing expression of osteoprotegerin, influencing the receptor activator of $\mathrm{NF}-x \mathrm{~B}$ ligand (RANKL)osteoprotegerin (OPG) pathway. Lovastatin is a natural statin which can be used to treat various diseases including atherosclerosis, sepsis, peripheral arterial disease, ischemic disease and bone fracture. Garrett and others (Garrett et al., 2007) reported that a high dose of statin given orally to femoral-fractured mice promoted fracture healing. However, statin given orally at a cholesterollowering dose does not reach the bones very well and only a minor fraction reaches the fracture site, so a high oral dose is required to achieve sufficient concentration of statin at the bone fracture site. However, high doses of statin can cause adverse effects such as liver failure, kidney disease and rhabdomyolysis. In order to achieve sufficient statin concentration at the fracture site without using high doses, it needs to be delivered directly to the fracture site (Bonnarens et al., 1984).

Similar type of study was carried out by Garrett and others (Garrett et al., 2007), who concluded that lovastatin administered in a nanobead preparation may be therapeutically useful in improving the healing of fractures in humans. Lovastatin nanobeads were able to stimulate bone formation in vitro at $5 \mathrm{ng} / \mathrm{ml}$ and increased rates of healing of femoral fractures upon a single drug injection into the fracture site, along with decreasing cortical fracture gap observed at 4 weeks with micro computed tomography. Ibrahim and others (Ibrahim et al., 2014) reported that combinatorial particulate delivery of lovastatin and tocotrienol directly to the fracture site could improve the strength of callus as well as mineralization during the fracture healing of osteoporotic bone. In our study, a single dose of lovastatin nanoparticles with the optimum drug concentration $(4 \mathrm{ng} / \mathrm{ml}$ ) markedly increased the strength and healing rate of the fractured bone. The increase in strength was observed to be dose dependent.

We conclude that lovastatin, when delivered through the controlled release formulation, effectively improves fracture healing in the rat model. The presented study provides evidence supporting the use of statins in the treatment of bone fractures. It also showed that statins may contribute to an increased osteogenesis and/or decreased bone resorption:

\section{Potential Conflict of Interest} ticle.

The authors declare no conflict of interest for this ar-

\section{REFERENCES}

Abdul Majeed S, Mohamed N, Soelaiman IN (2012) Effects of tocotrienol and lovastatin combination on osteoblast and osteoclast activity in estrogen deficient osteoporosis. Evid Based Complement Alternat Med 2012: 1-9. https:/ / doi.org/10.1155/2012/960742

Agnihotri SA, Mallikarjuna NN, Aminabhavi TM (2004) Recent advances on chitosan-based micro- and nanoparticles in drug delivery. J Control Release 100: 5-28. https://doi.org/10.1016/j.jconrel.2004.08.010

Altundag O, Altundag K, Selim Silay Y, Gunduz M, Demircan K (2004) Calcium and vitamin D supplementation during bisphos- 
phonate administration may increase osteoclastic activity in patients with bone metastasis. Med Hypotheses 63: https://doi.org/1010-1013. 10.1016/j.mehy.2004.04.022

Bhattarai N, Ramay HR, Chou SH, Zhang M (2006) Chitosan and lactic acid-grafted chitosan nanoparticles as carriers for prolonged drug delivery. Int J Nanomedicine 1: 181-187. PMCID: PMC2426789

Bonnarens F, Einhorn TA (1984) Production of a standard closed fracture in laboratory animal bone. J Orthop Res 2: 97-101. https:// doi.org/10.1002/jor.1100020115

Claes L, Recknagel S, Ignatius A (2012) Fracture healing under healthy and inflammatory conditions. Nat Rev Rheumatol 31: 133-143. https://doi.org/10.1038/nrrheum.2012.1

Einhorn TA (1995) Enhancement of fracture healing. J Bone Joint Surg Am 77: 940-956. PMID: 7782368

Einhorn TA (1998) The cell and molecular biology of fracture healing. Clin Orthop Relat Res 355 (Suppl): S7-S21. PMID: 9917622

Gan Q, Wang T (2007) Chitosan nanoparticles as protein delivery carrier-systematic examination of fabrication conditions for efficient loading and release. Colloids Surfaces B 59: 24-34. https://doi. org/10.1016/j.colsurfb.2007.04.009

Garrett IR, Gutierrez GE, Rossini G, Nyman J, McCluskey B (2007) Locally delivered lovastatin nanoparticles enhance fracture healing in rats. J Orthop Res 25: 1351-1357. https://doi.org/10.1002/jor.20391

Gerstenfeld LC, Cullinane DM, Barnes GL, Graves DT, Einhorn TA (2003) Fracture healing as a postnatal developmental process: molecular, spatial, and temporal aspects of its regulation. J Cell Biochem 88: 873-884. https://doi.org/10.1002/jcb.10435

Giannotti S, Bottai V, Dell'osso G, Pini E, De Paola G, Bugelli G, Guido G. (2013) Current medical strategies concerning fracture healing. 2013. Clin Cases Mineral Bone Metabolism 10: 116-120. PMCID: PMC3796998

Grundnes O, Reikeras O (1993) The importance of the hematoma for fracture healing in rats. Acta Orthop Scand 64: 340-342. https://doi. org/10.3109/17453679308993640

Gupta RB (2006) Fundamentals of drug nanoparticles. In Nanoparticle Technology for Drug Delivery. Gupta RB, Kompella UB, eds. pp 1-18. New York: Taylor \& Francis Group.

Hadjiargyrou M, Lombardo F, Zhao S, Ahrens W, Joo J, Ahn H, Jurman M, White DW, Rubin CT (2002) Transcriptional profiling of bone regeneration. Insight into the molecular complexity of wound repair. I Biol 277: 30177-30182. https://doi.org/10.1074/jbc. M203171200

Hausman MR, Schaffler MB, Majeska RJ (2001) Prevention of fracture healing in rats by an inhibitor of angiogenesis. Bone 29: 560-564. PMID: 11728927

Ibrahim N, Khamis MF, Mod Yunoh MF, Abdullah S, Mohamed N, Shuid AN (2014) Targeted delivery of lovastatin and tocotrienol to fracture site promotes fracture healing in osteoporosis model: micro computed tomography and biomechanical evaluation. PLOS ONE 9: e115595. https://doi.org/10.1371/journal.pone.0115595

McAnally JA, Gupta J, Sodhani S, Bravo L, Mo H (2007) Tocotrienols potentiate lovastatin mediated growth suppression in vitro and in vivo. Exp Biol Med 232: 523-531. PMID: 17392488

Mohammadpour Dounighi N, Eskandari R, Avadi MR, Zolfagharian H, Mir Mohammad Sadeghi A, Rezayat M (2012) Preparation and in vitro characterization of chitosan nanoparticles containing Mesobuthus eupeus scorpion venom as an antigen delivery system. $J$ Venom Anim Toxins Incl Trop Dis 18: 44-52

Rezaei Mokarram A, Alonso MJ (2006) Preparation and evaluation of chitosan nanoparticles containing diphtheria toxoid as new carriers for nasal vaccine delivery in mice. Arch Razi Inst 61: 13-25

Stürmer EK, Seidlová-Wuttke D, Sehmisch S, Rack T, Wille J, Frosch KH, Wuttke W, Stürmer KM (2006) Standardized bending and breaking test for the normal and osteoporotic metaphyseal tibias of the rat: effect of estradiol, testosterone, and raloxifene. J Bone Mineral Res 21: 89-96. https://doi.org/10.1359/JBMR.050913

Takahashi K, Setoguchi T, Tawaratsumida H, Arishima Y, Tominaga H, Ishidou Y, Nagano S, Shigemizu S, Aoki N, Akimoto M, Otsubo H, Matsuda T, Kakoi H, Izumi T, Nakamura S, Yokouchi M, Sunahara N, Komiya S (2015) Risk of low bone mineral density in patients with rheumatoid arthritis treated with biologics. BMC Musculoskelet Disord 16: 269. https://doi.org/10.1186/s12891-015-0732-x

World Health Organisation (2003) The burden of musculoskeletal conditions at the start of the new millennium. Report of a WHO Scientific Group.Technical Report Series. WHO, Geneva, Switzerland 919.

Yee CS, Xie L, Hatsell S, Hum N, Murugesh D, Economides AN, Loots GG, Collette NM (2015) Sclerostin antibody treatment improves fracture outcomes in Type I diabetic mouse model. Bone 82: 122-134. https://doi.org/10.1016/j.bone.2015.04.048 